\title{
Multiplicity of Herbig Ae/Be Stars
}

\author{
Sandrine J. Thomas ${ }^{1,2}$, Nicole S. van der Bliek ${ }^{2}$, Bernadette \\ Rodgers $^{3}$, Greg Doppmann ${ }^{3}$ and Jérôme Bouvier ${ }^{4}$ \\ ${ }^{1}$ LAO, UCO/Lick Observatory, 1156 High Street, Santa Cruz, CA 95064, USA. \\ email: sthomas@ucolick.org \\ ${ }^{2}$ NOAO/CTIO, Casilla 603, La Serena, Chile \\ email: nvdbliek@ctio.noao.edu \\ ${ }^{3}$ Gemini Observatory, Casilla 603, La Serena, Chile \\ email: brodgers@gemini.edu,gdoppmann@gemini.edu \\ ${ }^{4}$ University of Grenoble, BP53, F 38041, Grenoble, Cedex 9, France \\ email: Jerome.Bouvier@obs.ujf-grenoble.fr
}

\begin{abstract}
One of the most interesting constraints on star formation models comes from the study of multiplicity of young stars as a function of mass. While multiplicity studies of lowmass $\mathrm{T}$ Tauri stars have been quite exhaustive, an unbiased and systematic investigation of multiplicity among intermediate-mass Herbig Ae/Be (HAEBE) stars is still lacking. We are therefore conducting a photometric and spectroscopic survey of HAEBE stars to detect companions, establish their physical association with the primary and investigate their properties. The frequency and degree of multiplicity of HAEBE systems will provide new constraints on their formation mechanisms. In this paper we present preliminary results of the high resolution imaging part of the survey, carried out with the adaptive optics system Altair-NIRI on Gemini North. Of 72 stars observed, we find 44 possible binaries or multiples, including at least 25 not previously known.
\end{abstract}

Keywords. Herbig Ae/Be stars, binaries, pre-main sequence

\section{Introduction}

Herbig Ae/Be (HAEBE) stars are pre main-sequence stars of intermediate mass (2 to 9 solar masses). With masses between those of low-mass $\mathrm{T}$ Tauri stars and high mass young stars, HAEBE stars fill an important parameter space in addressing the question of star formation as a function of mass. At the same time, it is very likely that stars of different mass actually form and evolve together, as many stars form in clusters. A promising approach to constrain star formation models is thus to investigate the frequency of multiple pre-main sequence stars and study their properties as a function of mass of the primary stars. Like their low mass counterparts, the T Tauri stars, HAEBE stars are often found in groups: e.g., Bouvier \& Corporon (2001) find that HAEBE stars have a binary fraction significantly higher than both field $\mathrm{G}$ dwarfs and low mass young stars (both of order 60\%), while Testi et al. (1997) find a correlation between stellar density and spectral type. However, there are few studies of multiplicity of HAEBE stars and usually based on relatively small samples. A comprehensive, unbiased survey of the immediate environments of HAEBE stars is still lacking. We are therefore conducting a photometric and spectroscopic survey of HAEBE stars to detect multiple systems and to investigate the nature of the companions. The sample consists of all sources listed in Tables 1-3 of Thé et al. (1994) supplemented with HAEBE stars from the literature. This survey will provide us with a statistically meaningful data set on multiplicity of HAEBE stars. 
Here we present the first results of high resolution imaging observations, using AltairNIRI at Gemini North (program GN-2005B-Q-81), to search for companions to HAEBE stars.

\section{High resolution near infrared imaging}

Our total sample consists of more than 300 HAEBE stars with spectral types ranging from B to F selected from Tables 1-3 of Thé et al. (1994), supplemented with HAEBE stars listed in the literature. Previous studies found that visual companions can be 3 to 5 magnitudes fainter than the primaries [Leinert et al. (1997), Testi et al. (1997)]. To detect such faint companions at separations of less than $1^{\prime \prime}$, we obtained high resolution near infrared (NIR) $K$-band images, using adaptive optics systems on large telescopes: Altair-NIRI at Gemini North and NACO at the VLT. Here we present the results of the observations taken with Altair-NIRI at Gemini North, during the period September 2005 until March 2006. The stars in our sample have $K$ magnitudes ranging from $K=4$ to $K=12$, and they are bright enough to be used as guide stars themselves. Depending on the brightness of the source and on the observing conditions, the stars were observed either with the broad-band K-short filter or with a narrow-band $K$ filter centered at $\operatorname{Br} \gamma$. The field of view is $11^{\prime \prime}$ and the pixel size is 21.77 mas. Finally, we used the targets that did not have a (resolved) companion as reference point spread functions (PSF).

The data were reduced in a standard way (dark subtraction, flatfielding, sky substraction) with the IRAF Gemini package niri. In the case of relatively wide companions the astrometry and relative photometry were obtained by PSF fitting of the reference star, using an IDL version of the DAOPHOT PSF fitting procedure. For closer companions a PSF reconstruction method following Tokovinin et al. (2006) was used. Fig. 1 shows an example: HD 37411, where the system is actually a triple star or more.

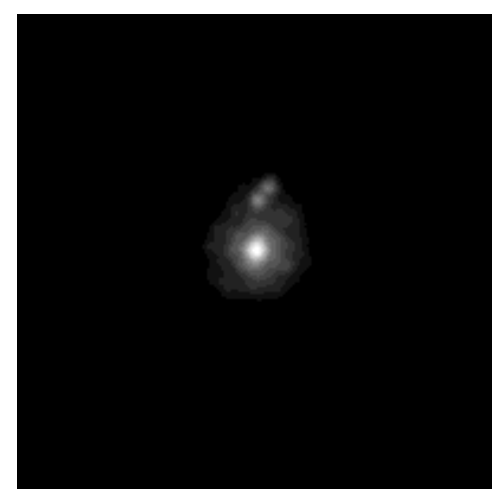

Figure 1. Example of a discovery, HD 37411.

We determined the detection limit of the system to check the reliability of our discoveries. This limit is defined as the minimum difference of magnitude $\Delta K$ observable at a given distance from the primary. First, the intensity radial variance $\sigma_{v a r}$ of the image centered on the primary is calculated. Then, the detection limit is set such that the minimum intensity increase due to the presence of a companion is greater than $5 \sigma_{\text {rad }}$.

Due to seeing variations, this detection limit has been calculated for each night of observation. However, the conditions of observation were chosen to be very similar for each night and therefore we could calculate a fairly accurate model shown on Fig. 2. 


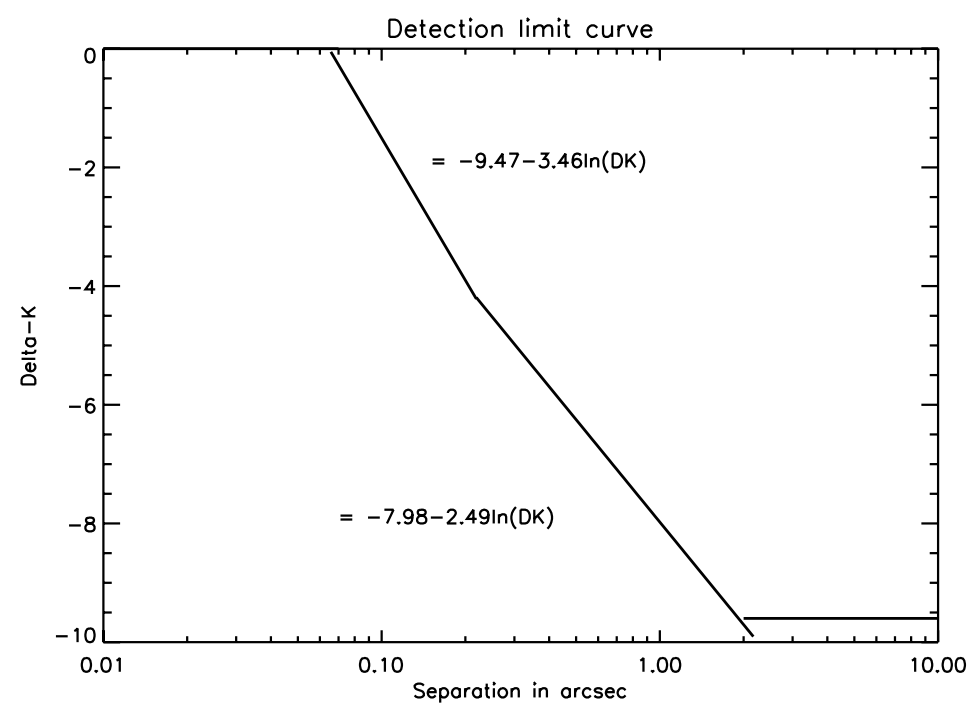

Figure 2. Detection limit for Altair.

\section{Preliminary results \& future work}

Of the 72 sources observed with Altair-NIRI during the period from September 2005 to March 2006, 44 potential binaries were found, of which 31 are not previously listed in the literature and 3 are ambiguous (may be artifacts). Not including these 3 , this brings the total number of HAEBE binary candidates to 66, nearly doubling the previously known sample. Table 1 lists the data of the binary candidates observed with AltairNIRI, regardless of their separation.

Of the 44 HAEBE binaries observed, 23, or $50 \%$, are potentially multiples rather than binaries, suggesting a preliminary binary fraction of HAEBE stars greater than 1 . In Table 1 sources with more than one possible companion are marked with an asterisk.

Table 1. Multiplicity. The targets with ${ }^{*}$ have more than one potential companion detected; with ${ }^{1}$ are the new discoveries; with ${ }^{2}$ are stars included in other studies but not identified as binaries. The 3 targets with (?) are ambiguous ones.

\begin{tabular}{|c|c|c|c|c|c|c|c|c|c|c|c|}
\hline Target & $\rho$ & $\theta$ & $\Delta \mathrm{m}$ & Target & $\rho$ & $\theta$ & $\Delta \mathrm{m}$ & Target & $\rho$ & $\theta$ & $\Delta \mathrm{m}$ \\
\hline PS Cep*1 & 6.66 & 310.1 & 7.9 & V1493 Cyg ${ }^{* 1}$ & 0.58 & 294.8 & 3.2 & VX Cas*2 & 5.34 & 165.3 & \\
\hline $\mathrm{V} 1429 \mathrm{Aql}^{* 1}$ & 1.19 & 319.3 & 7.4 & V1982 Cyg*1 & 2.01 & 302.1 & 7.7 & V628 Cas* & 0.76 & 269.6 & \\
\hline LkHA $168^{* 1}$ & 0.31 & 208.9 & 2.6 & HD $37258^{1}$ & 0.99 & 30.3 & 2.8 & V633 Cas & 5.78 & 4.4 & \\
\hline V751 Cyg*1 & 0.67 & 295.0 & 2.7 & BD-06 $1259^{1}$ & 2.04 & 46.7 & 4.0 & V1578 Cyg* & 1.31 & 309.6 & \\
\hline HD $235495^{* 1}$ & 2.08 & 297.0 & 4.6 & V1977 Cyg ${ }^{2}$ & 4.75 & 54.5 & 6.6 & IL Cep* & 7.80 & 329.2 & \\
\hline HBC $531^{* 1}$ & 6.61 & 163.0 & 5.7 & WW $\mathrm{Vul}^{2}$ & 7.29 & 239.7 & 6.5 & V700 Mon* & 0.15 & 55.6 & \\
\hline HBC $324^{* 1}$ & 8.01 & 354.0 & 3.9 & V374 $\mathrm{Cep}^{2}$ & 6.17 & 29.2 & 8.3 & XY Per & 1.33 & 256.7 & \\
\hline HD $37411^{* 1}$ & 0.46 & 357.5 & 3.3 & VV Ser ${ }^{2}$ & 7.47 & 249.6 & 7.1 & V892 Tau & 3.91 & 23.0 & \\
\hline V350 Ori $^{1}$ & 0.29 & 206.8 & 3.2 & HD $179218^{*} 2$ & 2.54 & 140.5 & 6.6 & HK Ori* & 0.35 & 40.8 & \\
\hline V385 Cep*1 & 2.84 & 229.9 & 7.9 & V699 Mon $^{2}$ & 8.50 & 226.6 & 4.8 & Mon & 0.71 & 293.6 & \\
\hline HD $245906^{1}$ & 0.13 & 77.1 & 1.5 & V1271 Ori $^{2}$ & 8.38 & 294.7 & 6.7 & BD-06 1253 & 0.12 & 232.8 & 1.4 \\
\hline LkHA $201^{* 1}$ & 2.34 & 325.8 & 6.2 & $\mathrm{CQ} \mathrm{Tau}^{2}$ & 2.09 & 55.5 & 8.5 & $\operatorname{HBC} 334^{*}(?)$ & 0.44 & 347 & \\
\hline HBC $535^{* 1}$ & 3.67 & 221.3 & 7.1 & V590 Mon ${ }^{2}$ & 5.07 & 97.1 & 6.6 & V390 Cep(?) & 0.30 & 248.8 & \\
\hline V1685 $\mathrm{Cyg}^{1}$ & 0.72 & 175.1 & 5.4 & HD $36112^{2}$ & 2.28 & 311.3 & 8.3 & VY Mon(?) & 2.05 & 240.7 & \\
\hline LkHA $147^{* 1}$ & 1.63 & 340.7 & 3.0 & HD $37357^{2}$ & 0.14 & 226.3 & 1.7 & & & & \\
\hline
\end{tabular}




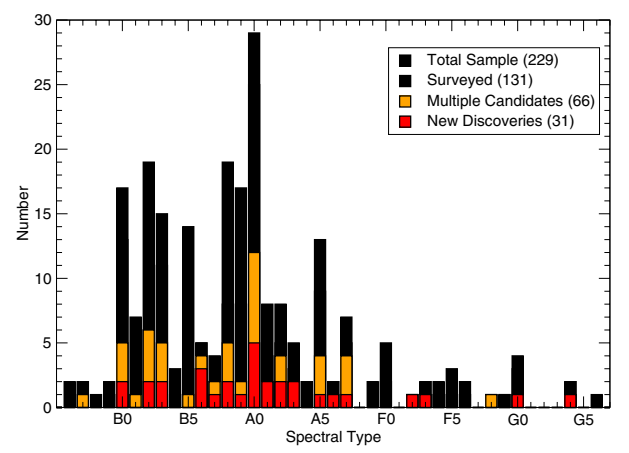

Figure 3. Multiplicity as a function of spectral type. Only stars with well-determined spectral types are included.

Fig. 3 shows multiplicity as a function of spectral type for our sample so far. The detection of possible companions around $50 \%$ of the stars surveyed does not appear to be a strong function of spectral type. However, more data are needed to draw significant conclusions.

The next step will be to establish physical association of the companions for which we will use NIR photometry and spectroscopy. For true companions we will determine spectral types and look for evidence of pre-main sequence status, either emission lines or near-infrared excess indicating the presence of circumstellar disks. We also plan to determine rotational and radial velocities. The final goals are to understand the nature of the companion stars and the effect of the nearby primary on their formation, and to study the multiplicity of HAEBE stars as function of mass.

\section{Acknowledgements}

We are thankful to Maria-José Cordero, an undergraduate student from the program PIA (Prácticas de Investigación en Astronomía) in Chile, who worked on the first results of the data set. Based on observations obtained at the Gemini Observatory, which is operated by the Association of Universities for Research in Astronomy, Inc., under a cooperative agreement with the NSF on behalf of the Gemini partnership: the National Science Foundation (United States), the Particle Physics and Astronomy Research Council (United Kingdom), the National Research Council (Canada), CONICYT (Chile), the Australian Research Council (Australia), CNPq (Brazil) and CONICET (Argentina).

\section{References}

Bouvier J. \& Corporon P. 2001, IAUS 200, 155

Leinert C., Richichi A., \& Haas M. 1997, A\&A 318, 472

Testi L., Palla F., Prusti T., Natta A., \& Maltagliati S. 1997, A\&SA 320, 159

Tokovinin A., Thomas S., Sterzik M., \& Udry S. 2006, A\& $A$, 450, p681

Thé, P. S., de Winter D., \& Perez M. R.1994 A\&AS 104, 315 